\title{
Antiparasitic resistance
}

\section{KEY POINTS}

- Practitioners should regularly review the current status of resistance to companion animal antiparasitics

- Control of parasites should be practiced through responsible product use

- There should be a planned approach to apparent efficacy failure

- Any suspected resistance should be reported under the Suspected Adverse Reaction Reporting Scheme (SARRS)

In theory, any of the ectoparasites (fleas, ticks, lice, flies, sandflies and mites) or the gastrointestinal helminths (including roundworms and tapeworms) challenging domestic pets could develop resistance to the antiparasitic medicines used to control them, although there is a consensus that resistance at present is less likely in tapeworms with their more complex two-host lifecycle.

A current definition of antiparasitic resistance states: 'the selection of a specific heritable trait (or traits) in a population of parasites due to that population's contact with a chemical that results in a significant increase in the percentage of the population that will survive a standard dose of that chemical' (Coles and Dryden, 2014). It should be noted that, within parasite populations, there may be considerable natural variation in susceptibility.

\section{Evidence of resistance to companion animal antiparasitics}

To date, treatment failure of authorized antiparasitic preparations used according to product-approved label directions caused by resistant parasitic arthropods has not been proven in the UK or EU. However, flea Ctenocephalides felis gene mutations associated with resistance to dieldrin ( $\mathrm{rdl}$ ) and knock down resistance (kdr) and super knock down resistance (skdr) have been identified in UK flea populations. The implications of these mutations for insecticide efficacy remain unclear. Reports exist from the USA of fleas developing resistance to many older classes of insecticide. Reduced efficacy against fleas recently seen with a fipronil-methoprene combination may be the result of resistance, innately tolerant flea strains or other unknown factors (Dryden et al., 2013). There are a few reports of individual tick populations, particularly the brown tick Rhipicephalus sanguineus developing acaricidal resistance on the American continent (Coles and Dryden, 2014).

There have been few proven cases of anthelmintic resistance (AR) in worms from cats and dogs. Resistance to pyrantel and to benzimidazoles has been reported in the dog hookworm, Ancylostoma caninum, in Australia and Brazil, respectively (Kopp et al., 2007; Furtado et al., 2014). Macrocyclic lactone heartworm preventives in both the USA and Europe aim to achieve $100 \%$ prevention of the development of adult Dirofilaria immitis heartworms. In the USA (but not Europe) there have been a number of protection failures where the worms have characteristic genotypic changes that differentiate them from susceptible isolates (Bourguinat et al., 2017).

Development of resistance to a variety of anti-protozoals, including metronidazole, can occur with Giardia spp., which has to be distinguished from other causes of treatment failure including reinfection (Fiechter et al., 2012).

\section{Parasite control and efficacy monitoring}

Whilst helminth control in horses includes leaving some animals with low level infections untreated in refugia to reduce selection pressure on the whole population, this concept does not translate readily into companion animal parasite control, where there exists a public expectation for zero tolerance of parasites, particularly where there is a zoonotic risk. Therefore, alternative strategies must be sought.

Parasiticide mixes (a range of possible scenarios, including fixed combinations and sequential administration of licensed products over a period of time) and rotation potentially have value in delaying or preventing resistance, particularly in combination with management strategies. Whilst these principles have been developed for crop pests, and Control of Worms Sustainably (COWS) and Sustainable Control of Parasites (SCOPS) have developed guidance for anthelmintic use in cattle and sheep respectively, more work is needed on their applicability to pet parasites.

Monitoring is important, especially in kennels and catteries, where selection pressure may be particularly high if the same parasiticide is used repeatedly over time. The special considerations associated with rescue kennels have recently been considered in detail by Raza et al., 2018.

Timely and effective control of parasite infections, thereby preventing them from becoming an ongoing problem, is important. Ectoparasiticides aim to eliminate existing pet parasite infestations and, with fleas, their environmental stages. 
Control of flea environmental stages can include not only chemical treatment of the pet's bedding and household carpets, but also areas the pet frequents (e.g. the shed or family car). Vacuuming home carpets plus washing pets' bedding are important components in flea management. Success of the strategy also relies on all household pets being treated simultaneously; identifying and eliminating flea infestation 'hot spots'; ensuring that the family pet is not exposed to other parasite infested animals or contaminated environments outside the household; and recognizing that shampooing or swimming may decrease the effectiveness of topical products.

All major worms (excluding heartworm) are transmitted by the passage of eggs or larvae in faeces, hence hygiene measures, especially cleaning up pet faeces regularly, will reduce environmental contamination with infective parasite stages and assist in control alongside parasiticide use.

In the case of heartworm, it is important to check that the pet does not already have adult heartworms prior to commencing preventive treatment, as it is suspected that exposure of adult worms and microfilariae to preventives may be implicated in resistance development in the USA (Bowman, 2012). In addition, there are also concerns regarding target animal safety with inadvertently administering certain preventative products to animals with circulating microfilaria.

\section{Investigation and management of suspected resistance}

When treatment failure may be associated with parasite resistance, it is important to carry out a systemic investigation to rule out non-compliance and high environmental challenge. Initial checks to confirm that the prescribed control strategy was applied as directed should be carried out. A 2012 survey of dog owners using monthly spot-on tick treatments found that these products were not used as recommended in $56 \%$ of dogs (Beck et al., 2013) and when control measures were properly and consistently applied $92 \%$ of households that had experienced a flea control problem were cleared of fleas. Conducting a successful investigation relies on the relationship between clinician and client. An open relaxed relationship built on trust and respect goes a long way to understanding the client's compliance behaviour in relation to parasite control.

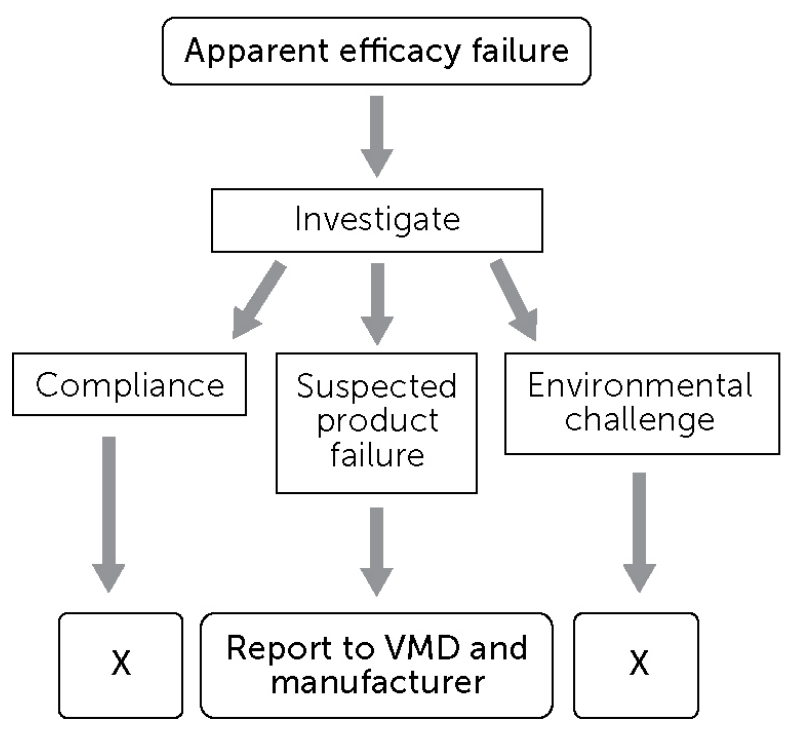

\section{Questions to be asked of the client when suspecting product failure relating to flea control}

Did the pet owner:
1. Comply with the product treatment regimen?
2. Use commercial chemical treatments for pet bedding,
household carpets, the family car and garden shed?
3. Vacuum the household carpet?
4. Maintain environmental hygiene relative to the pet?
5. Wash the pet bedding?
6. Restrict pet access only to other treated animals?
7. Restrict pet access to contaminated environments
outside the household including catteries and kennels?
Ensure that a pet treated with a topical parasiticide is
not inappropriately shampooed or permitted near
9. Maintain a record of the travel history of the pet
including an up to date pet passport?

In vitro tests exist for insect and tick strains to establish their susceptibility to parasiticides. At present, there is no way of detecting AR in dogs and cats other than by the faecal egg count reduction test, which is time-consuming and labour intensive, requiring the pet owner's cooperation. When investigations lead to suspect product failure related to AR in worms, issues such as compliance with the product treatment regime, environmental hygiene, feeding of non-commercial diets, contact with other animals, exposure to kennel/cattery environments and access to rodents and slugs, along with the travel history of the pet, need to be considered.

When unmet expectations and non-compliance have been eliminated and resistance remains a concern, then it is important to report the suspected lack of efficacy to the Veterinary Medicines Directorate (VMD) or to the marketing authorization holder (MAH) so that they can investigate the problem quickly. Unfortunately, as has been documented, owners may be keen to eliminate the problem, thus destroying material for any future investigation.

\section{References}

- Beck S, Schein E, Baldermann C, von Samson-Himmelstjerna G and Kohn B (2013) Tick infestation and tick prophylaxis in dogs in the area of Berlin/Brandenburg - results of a questionnaire study. Berliner und Münchener Tierärztliche Wochenschrift 126, 67-76

- Bourguinat C, Lefebvre F, Sandoval J et al. (2017) Dirofilaria immitis JYD-34 isolate: whole genome analysis. Parasites \& Vectors 10, 494

- Bowman DD (2012) Heartworms, macrocyclic lactones, and the spectre of resistance to prevention in the United States. Parasites \& Vectors 5, 138

- Coles TB and Dryden MW (2014) Insecticide/acaricide resistance in fleas and ticks infecting dogs and cats. Parasites 8 Vectors 7,8 
- Dryden MW, Payne PA, Smith V et al. (2013) Evaluation of indoxacarb and fipronil (s)-methoprene topical spot-on formulations to control flea populations in naturally infested dogs and cats in private residences in Tampa Fl. USA. Parasites \& Vectors 6, 366

- Fiechter RM-E, Deplazes P and Schnyder M (2012) Control of Giardia infections with ronidazole and intensive hygiene management in a dog kennel. Veterinary Parasitology 187, 93-98

- Furtado LF, Bello AC, dos Santos HA et al. (2014) First identification of the F200Y SNP in the $\beta$-tubulin gene linked to benzimidazole resistance in Ancylostoma caninum. Veterinary Parasitology 206, 313-316

- Kopp SR, Kotze AC, McCarthy JS et al. (2007) High-level pyrantel resistance in the hookworm Ancylostoma caninum Veterinary Parasitology 143, 299-304

- Raza A, Rand J, Ghaffar Qamar A, Jabbar A and Kopp S (2018) Gastrointestinal Parasites in Shelter Dogs: Occurrence, Pathology, Treatment and Risk to Shelter Workers. Animals 8, 108

\section{Useful websites}

Control of Worms Sustainably (COWS) (@)

Sustainable Control of Parasites (SCOPS) (i)

\section{QUESTIONS}

1. It is generally considered that the following pet parasites are least likely to develop resistance:
a. Tapeworms
b. Heartworms
c. Fleas
d. Ticks

2. Resistance prevention and control strategies have been well developed for:
a. Veterinary parasiticides
b. Crop protection products
c. Human parasiticides
d. Tapeworms

3. Fleas in the UK have been identified with which gene mutations?
a. Rdl, kdr and skdr
b. Mdr, kdr and skdr
c. Kdr, skdr and rdr
d. Rdl, kdl and sdl

4. Which one of the following options is most likely to cause apparent flea treatment failure?
a. Treating only some of the animals in the household
b. Treating with an adulticide
c. Treating with an environmental treatment
d. Hoovering daily

5. In the USA heartworm reduced preventive efficacy has been reported to:
a. Macrocyclic lactones
b. Pyrantel
c. Diethylcarbamazine
d. Benzimidazoles 